\title{
BOUNDEDNESS PROPERTIES IN VOLTERRA INTEGRODIFFERENTIAL SYSTEMS
}

\author{
W. E. MAHFOUD
}

ABSTRACT. Sufficient conditions are given to insure that all solutions of the integrodifferential system

$$
x^{\prime}=A(t) x+\int_{0}^{t} C(t, s) x(s) d s+f(t)
$$

are uniform bounded.

1. Introduction. Consider the integrodifferential systems

$$
x^{\prime}=A(t) x+\int_{0}^{t} C(t, s) x(s) d s+f(t)
$$

and

$$
y^{\prime}=A(t) y+\int_{0}^{t} C(t, s) y(s) d s,
$$

where $A(t)$ and $C(t, s)$ are continuous $n \times n$ matrices, $n \geq 1,0 \leq s \leq t<\infty$, and $f:[0, \infty) \rightarrow R^{n}$ is continuous.

For any $t_{0} \geq 0$ and any continuous function $\phi:\left[0, t_{0}\right] \rightarrow R^{n}$, a solution of (1) is a function $x:[0, \infty) \rightarrow R^{n}$ satisfying (1) for $t \geq t_{0}$ and such that $x(t)=\phi(t)$ for $0 \leq t \leq t_{0}$. Under the stated conditions, (1) has a unique solution denoted by $x\left(t, t_{0}, \phi\right)$ or simply $x(t)$ if no confusion should arise. The solution $x(t) \equiv 0$ of $(2)$ is called the zero solution. Solutions of (1) satisfying $x(0)=x_{0}$ are given by the variation of parameters formula

$$
x(t)=R(t, 0) x_{0}+\int_{0}^{t} R(t, s) f(s) d s,
$$

where $R(t, s)$ is the $n \times n$ matrix satisfying

$$
\frac{\partial R(t, s)}{\partial s}=-R(t, s) A(s)-\int_{s}^{t} R(t, u) C(u, s) d u, \quad R(t, t)=I
$$

(see Grossman and Miller [4]). If $D$ is a matrix or a vector, $|D|$ means the sum of the absolute values of its elements.

Formula (3) plays a central role in perturbation theory. Suppose for instance that $\int_{0}^{t}|R(t, s)| d s \leq M$ for some positive constant $M$; if $f$ is bounded and solutions of $(2)$ are bounded, then every solution $x\left(t, 0, x_{0}\right)$ of $(1)$ is bounded. On the other hand if solutions of (1) are bounded for every bounded $f$, then $R(t, 0)$ and $\int_{0}^{t} R(t, s) f(s) d s$ are bounded, and by an argument parallel to the one used in

Received by the editors February 19, 1986.

1980 Mathematics Subject Classification (1985 Revision). Primary 45D05; Secondary 34K20. 
the proof of Perron's theorem [5, pp. 152-153], $\int_{0}^{t}|R(t, s)| d s$ is bounded. Thus, information such as boundedness of solutions of (1) leads to information about $R(t, s)$ which can be used in conjunction with (3) to yield perturbation results for nonlinear systems. For detailed discussion and further results concerning $R(t, s)$, see Grimmer and Seifert [3]. Also, the property of boundedness of solutions of (1) together with the asymptotic stability of the zero solution of (2) yield existence of periodic solutions of some limiting systems (Burton [2]).

The purpose of this paper is to give sufficient conditions to insure that (1) has bounded solutions. Results of this sort can be found in [3 and 1], but their applicability is limited by the conditions on $A(t)$ and $C(t, s)$. For instance, in [3], it is assumed that $A(t)$ is constant and stable unless $C(t, s)=C(t-s)$. In [1], $A(t)$ must be stable unless $n=1$ and $A(t)$ is a nonconstant function. Our results here are more general and apply to $(1)$ whether $A(t)$ is stable, identically zero, or completely unstable, and do not require $A(t)$ to be constant nor $C(t, s)$ to be a convolution kernel. Furthermore, the technique is new and the main result unifies, improves, and extends major results in [1 $\mathbf{1}$ and $\mathbf{3}]$.

For convenience, we state below some relevant results in [3 and 1]. A major result in $[3]$ is Theorem 4; we state only the main part, the rest follows from (3) and (4) as discussed above.

ThEOREM A (GRIMMER-SEIFERT). Suppose $A(t)=A=$ constant and $A$ is a stable matrix. Let $D$ be the positive definite symmetric matrix satisfying $A^{T} D+$ $D A=-I$, and suppose there is a positive constant $M$ such that

$$
\int_{0}^{t}|D C(t, s)| d s \leq M<\alpha /(2 \beta),
$$

where $\alpha^{2}$ and $\beta^{2}$ are respectively the smallest and largest eigenvalues of $D$. Then if $f$ is bounded, all solutions of (1) are bounded.

The case $n=1$ is the most interesting in $[\mathbf{1}]$ as $A(t)$ can take some nonnegative values. We state the essential findings from $[1, \mathrm{pp} .170-172]$ in

THEOREM B (BURTON). Suppose $n=1$ and there exist continuous functions $\alpha:[0, \infty) \rightarrow R$ and $\Phi(t, s) \geq 0$, and positive constants $b, M$, and $P$ such that

(i) $\alpha(t) \leq M$,

(ii) $A(t)+\alpha(t) \leq-b$,

(iii) $\alpha(t) e^{-b(t-s)}-\int_{s}^{t} e^{-b(t-u)}|C(u, s)| d u \geq-\Phi(t, s)$,

(iv) $\int_{0}^{t} \Phi(t, s) d s \leq P<1$, and

(v) $\int_{0}^{t} e^{-b(t-s)}|f(s)| d s \leq M$.

Then solutions of (1) are uniform bounded, and the zero solution of (2) is uniformly stable. 6].

For the various stability definitions used here, we refer the reader to $[\mathbf{1}, \mathbf{3}$, and

2. Main results. Let $B(t)$ and $K(t, s)$ be continuous $n \times n$ matrices, $0 \leq s \leq$ $t<\infty$. Following Hale's notation [5], for every $s \in[0, \infty)$, we let $Z(t, s)$ denote the $n \times n$ matrix satisfying

$$
Y^{\prime}=B(t) Y, \quad Y(s)=I .
$$


Let $H(t, s)$ be the $n \times n$ matrix satisfying

$$
\partial H(t, s) / \partial t=B(t) H(t, s)+K(t, s), \quad H(s, s)=A(s)-B(s) .
$$

Then

$$
H(t, s)=Z(t, s)[A(s)-B(s)]+\int_{s}^{t} Z(t, u) K(u, s) d u
$$

Let

$$
V(t, x(\cdot))=x(t)-\int_{0}^{t} H(t, s) x(s) d s .
$$

The derivative of $V(t, x(\cdot))$ along a solution $x(t)=x\left(t, t_{0}, \phi\right)$ of $(1)$ satisfies

$$
\begin{aligned}
V^{\prime}(t, x(\cdot)) & =x^{\prime}-H(t, t) x-\int_{0}^{t}[\partial H(t, s) / \partial t] x(s) d s \\
& =[A(t)-H(t, t)] x+\int_{0}^{t}[C(t, s)-\partial H(t, s) / \partial t] x(s) d s+f(t) .
\end{aligned}
$$

By (7) and (9)

$$
V^{\prime}(t, x(\cdot))=B(t) V(t, x(\cdot))+\int_{0}^{t}[C(t, s)-K(t, s)] x(s) d s+f(t) .
$$

Thus

$$
V(t, x(\cdot))=Z\left(t, t_{0}\right) V\left(t_{0}, \phi\right)+\int_{t_{0}}^{t} Z(t, u) g(u, x(\cdot)) d u
$$

where

$$
g(t, x(\cdot))=\int_{0}^{t}[C(t, s)-K(t, s)] x(s) d s+f(t) .
$$

THEOREM 1. Let $Z(t, s)$ be the solution of (6), and suppose there are positive constants $N, J$, and $M$ such that

(i) $\left|Z\left(t, t_{0}\right)\right| \leq N$,

(ii) $\int_{0}^{t}\left|Z(t, s)[A(s)-B(s)]+\int_{s}^{t} Z(t, u) C(u, s) d u\right| d s \leq J<1$, and

(iii) $\left|\int_{t_{0}}^{t} Z(t, u) f(u) d u\right| \leq M$.

Then all solutions of (1) are uniform bounded, and the zero solution of (2) is uniformly stable.

Proof. Let $K(t, s)=C(t, s)$. Then by (8), (ii) is precisely $\int_{0}^{t}|H(t, s)| d s \leq$ $J<1$. If $\|\phi\|=\max _{0 \leq t \leq t_{0}}|\phi(t)|$, then by (9), (11) and (i)-(iii),

$$
\begin{aligned}
|V(t, x(\cdot))| & \leq\left|Z\left(t, t_{0}\right)\right|\left|V\left(t_{0}, \phi\right)\right|+\left|\int_{t_{0}}^{t} Z(t, u) f(u) d u\right| \\
& \leq(1+J) N\|\phi\|+M .
\end{aligned}
$$

If $\|\phi\|<B_{1}$ for some constant $B_{1}$ and if $K=2 N B_{1}+M$, then

$$
|x(t)|-\int_{0}^{t}|H(t, s)||x(s)| d s \leq|V(t, x(\cdot))| \leq K .
$$


Now, either there is a $B_{2}>0$ such that $|x(t)|<B_{2}$ for all $t \geq t_{0}$, and this is uniform boundedness, or there is a monotone sequence $\left\{t_{n}\right\}$ tending to $\infty$ such that $\left|x\left(t_{n}\right)\right|=\max _{0 \leq t \leq t_{n}}|x(t)|$ and $\left|x\left(t_{n}\right)\right| \rightarrow \infty$ as $t_{n} \rightarrow \infty$. By (ii) and (12),

$$
\left|x\left(t_{n}\right)\right|(1-J) \leq\left|x\left(t_{n}\right)\right|-\int_{0}^{t_{n}}\left|H\left(t_{n}, s\right)\right||x(s)| d s \leq K,
$$

a contradiction. This completes the proof.

REMARK 1. Condition (i) of Theorem 1 is exactly the statement that the system $y^{\prime}=B(t) y$ is uniformly stable. If $B(t)$ is selected so that $y^{\prime}=B(t) y$ is uniformly asymptotically stable (u.a.s.), then (ii) is also satisfied for every bounded $f$. If the system $z^{\prime}=A(t) z$ is stable, one chooses $B(t)=A(t)$. If $A(t)=A=$ constant, we take $B(t)=B=$ constant. In this case, $Z(t, s)=Z(t-s)$ and the statement that $y^{\prime}=B y$ is u.a.s. is equivalent to the statement that $B$ is a stable matrix; i.e. all eigenvalues of $B$ have negative real parts.

COROLlaRY 1. Suppose the system $z^{\prime}=A(t) z$ is u.a.s. If $f$ is bounded and

$$
\int_{0}^{t}\left|\int_{s}^{t} Z(t, u) C(u, s) d u\right| d s \leq J<1
$$

then all solutions of (1) are uniform bounded.

COROLlARY 2. If $n=1$ and $b, J$, and $M$ are positive constants such that

(i) $\int_{0}^{t}\left|[A(s)+b] e^{-b(t-s)}+\int_{s}^{t} e^{-b(t-u)} C(u, s) d u\right| d s \leq J<1$, and

(ii) $\left|\int_{0}^{t} e^{-b(t-u)} f(u) d u\right| \leq M$,

then all solutions of (1) are uniform bounded.

Proof. Let $B(t)=-b$. Then $Z(t, s)=e^{-b(t-s)}$ and

$$
\begin{aligned}
\left|\int_{t_{0}}^{t} Z(t, u) f(u) d u\right| & \leq\left|\int_{0}^{t} e^{-b(t-u)} f(u) d u\right|+\left|\int_{0}^{t_{0}} e^{-b(t-u)} f(u) d u\right| \\
& \leq M+e^{-b t_{0}}\left|\int_{0}^{t_{0}} e^{b u} f(u) d u\right| \leq 2 M
\end{aligned}
$$

Thus, (i)-(iii) of Theorem 1 are satisfied, and this completes the proof.

REMARK 2. In order to compare Theorem 1 and Theorem B, we first observe that (ii)-(iv) of Theorem B imply

$$
\int_{0}^{t}\left[(A(s)+b) e^{-b(t-s)}+\int_{s}^{t} e^{-b(t-u)}|C(u, s)| d u\right] d s \leq P<1 .
$$

If we multiply both sides by $e^{b t}$ and rearrange the terms, we obtain

$$
\int_{0}^{t} A(s) e^{b s} d s+\int_{0}^{t} \int_{s}^{t} e^{b u}|C(u, s)| d u d s \leq(P-1) e^{b t}+1 .
$$

Since $P<1$, the right side tends to $-\infty$ as $t \rightarrow \infty$, and hence $\int_{0}^{\infty} A(t) e^{b t} d t=-\infty$. Thus, Theorem $\mathrm{B}$ requires that $A(t)$ be strongly negative most of the time; in particular, when $A(t)=A=$ constant, it requires that $A$ be negative. In essence, Corollary 2 includes Theorem B and hence, Theorem 1 extends Burton's results to (1) where $A(t)$ may be identically zero or completely unstable.

The following example is given for illustration. 
EXAMPLE 1. Consider the scalar equation

$$
x^{\prime}=A(t) x-\left(\beta^{2} / 4\right) \int_{0}^{t} e^{-\beta(t-s)} \psi(t, s) x(s) d s+f(t),
$$

where $A, \psi$, and $f$ are continuous, $f$ is bounded, $0 \leq A(t) \leq 1,0<\alpha \leq \psi(t, s) \leq 1$, and $\alpha \beta>4$.

Let $b=\beta / 2$ in Corollary 2. Then

$$
\begin{aligned}
H(t, s) & =e^{-\beta(t-s) / 2}\left[A(s)+(\beta / 2)-\left(\beta^{2} / 4\right) \int_{s}^{t} e^{-\beta(u-s) / 2} \psi(u, s) d u\right] \\
& \leq e^{-\beta(t-s) / 2}\left[1+(\beta / 2)-(\alpha \beta / 2)+(\alpha \beta / 2) e^{-\beta(t-s) / 2}\right] .
\end{aligned}
$$

On the other hand,

$$
H(t, s) \geq e^{-\beta(t-s) / 2}\left[(\beta / 2)-\left(\beta^{2} / 4\right) \int_{s}^{t} e^{-\beta(u-s) / 2} d u\right]=(\beta / 2) e^{-\beta(t-s)} \geq 0 .
$$

Thus,

$$
\int_{0}^{t}|H(t, s)| d s \leq\left(\frac{2}{\beta}+1-\alpha\right)\left(1-e^{-\beta t / 2}\right)+\frac{\alpha}{2}\left(1-e^{-\beta t}\right) \leq \frac{2}{\beta}-\frac{\alpha}{2}+1<1 .
$$

By Corollary 2, all solutions are uniform bounded.

REMARK 3. In order to compare Theorem 1 and Theorem $\mathrm{A}$, we look at a special case of (1). Let $A(t)=A=$ constant and let $A$ be a stable diagonal matrix; i.e. $A=\left[-a_{i j}\right]$ with $a_{i j}>0$ if $i=j$ and $a_{i j}=0$ if $i \neq j$. The positive definite symmetric matrix $D=\left[d_{i j}\right]$ satisfying $A^{T} D+D A=-I$ is also diagonal with $d_{i i}=1 /\left(2 a_{i i}\right)$. For definiteness, we assume that $a_{11} \leq a_{i i} \leq a_{n n}, i=1,2, \ldots, n$, so that the smallest and largest eigenvalues of $D$ are $\alpha^{2}=1 /\left(2 a_{n n}\right)$ and $\beta^{2}=1 /\left(2 a_{11}\right)$. If $C(t, s)=\left[c_{i j}(t, s)\right], \gamma_{i}(t, s)=\sum_{j=1}^{n}\left|c_{i j}(t, s)\right|$, and $M_{i}=\sup _{t \geq 0} \int_{0}^{t} \gamma_{i}(t, s) d s$, then

$$
|D C(t, s)|=\sum_{i=1}^{n} \gamma_{i}(t, s) /\left(2 a_{i i}\right)
$$

and the integral condition of Theorem $\mathrm{A}$ reduces to

$$
\sum_{i=1}^{n} M_{i} / a_{i i}<\sqrt{a_{11} / a_{n n}}
$$

On the other hand, if $B(t)=A$ in (6), then the matrix $Z(t-s)$ is diagonal with $Z(t)=\left[e^{-a} i j^{t}\right]$, and

$$
\begin{aligned}
\int_{0}^{t} \mid & H(t, s)\left|d s=\int_{0}^{t}\right| \int_{s}^{t} Z(t-u) C(u, s) d u\left|d s \leq \int_{0}^{t} \int_{s}^{t}\right| Z(t-u) C(u, s) \mid d u d s \\
& =\int_{0}^{t} \int_{0}^{u}|Z(t-u) C(u, s)| d s d u=\sum_{i=1}^{n} \int_{0}^{t} \int_{0}^{u} e^{-a_{i i}(t-u)} \gamma_{i}(u, s) d s d u \\
& \leq \sum_{i=1}^{n} \int_{0}^{t} M_{i} e^{-a_{i i}(t-u)} d u \leq \sum_{i=1}^{n} M_{i} / a_{i i}
\end{aligned}
$$


The integral condition of Corollary 1 is satisfied if

$$
\sum_{i=1}^{n} M_{i} / a_{i i}<1 \text {. }
$$

It follows from (14) and (15) that, in this special case, (13) of Corollary 1 is milder than (5) of Theorem $\mathrm{A}$, and that, for $n=1$, Theorem 1 includes Theorem A. Furthermore, for $n>1$ and $\alpha / \beta$ very small, (5) can be severe. This is illustrated in the following example.

EXAMPLE 2. Consider the system

$$
x^{\prime}=A x+\int_{0}^{t} C(t, s) x(s) d s+f(t)
$$

where

$$
A=\left[\begin{array}{cc}
-1 & 0 \\
a-1 & -a
\end{array}\right], \quad a>1, \quad C(t, s)=\left[c_{i j}(t, s)\right],
$$

$c_{i j}(t, s), i, j=1,2$, are continuous, and $f$ is continuous and bounded.

The positive definite symmetric matrix $D$ satisfies $A^{T} D+D A=-I$ is given by

$$
D=\frac{1}{2 a(a+1)}\left[\begin{array}{cc}
2 a^{2}-a+1 & a-1 \\
a-1 & a+1
\end{array}\right]
$$

and

$$
\begin{aligned}
\int_{0}^{t}|D C(t, s)| d s= & \frac{a}{a+1} \int_{0}^{t}\left[\left|c_{11}(t, s)\right|+\left|c_{12}(t, s)\right|\right] d s \\
& +\frac{1}{a+1} \int_{0}^{t}\left[\left|c_{21}(t, s)\right|+\left|c_{22}(t, s)\right|\right] d s .
\end{aligned}
$$

Let $M_{i}=\sup _{t \geq 0} \int_{0}^{t}\left[\left|c_{i 1}(t, s)\right|+\left|c_{i 2}(t, s)\right|\right] d s, i=1,2$. Then (5) reduces to

$$
[a /(a+1)] M_{1}+[1 /(a+1)] M_{2}<\alpha /(2 \beta) .
$$

The eigenvalues $\alpha^{2}$ and $\beta^{2}$ of $D$ are solutions of the equation $\lambda^{2}-2\left(a^{2}+1\right) \lambda+$ $2 a\left(a^{2}+1\right)=0$ with $\alpha /(2 \beta)=\sqrt{a} /\left[\sqrt{2}\left(\sqrt{a^{2}+1}+a-1\right)\right]$ and $\alpha /(2 \beta) \rightarrow 0$ as $a \rightarrow \infty$.

On the other hand, with $B=A$, we have

$$
Z(t)=\left[\begin{array}{cc}
e^{-t} & 0 \\
e^{-t}-e^{-a t} & e^{-a t}
\end{array}\right]
$$

and

$$
\begin{aligned}
\int_{0}^{t}|H(t, s)| d s= & \int_{0}^{t}\left|\int_{s}^{t} Z(t-u) C(u, s) d u\right| d s \leq \int_{0}^{t} \int_{0}^{u}|Z(t-u) C(u, s)| d s d u \\
= & \int_{0}^{t} \int_{0}^{u}\left[2 e^{-(t-u)}-e^{-a(t-u)}\right]\left[\left|c_{11}(u, s)\right|+\left|c_{12}(u, s)\right|\right] d s d u \\
& +\int_{0}^{t} \int_{0}^{u} e^{-a(t-u)}\left[\left|c_{21}(u, s)\right|+\left|c_{22}(u, s)\right|\right] d s d u \\
\leq & M_{1} \int_{0}^{t}\left[2 e^{-(t-u)}-e^{-a(t-u)}\right] d u+M_{2} \int_{0}^{t} e^{-a(t-u)} d u \\
= & 2 M_{1}\left(1-e^{-t}\right)+(1 / a)\left(M_{2}-M_{1}\right)\left(1-e^{-a t}\right) .
\end{aligned}
$$


The right-hand side is an increasing function of $t$ which tends to $2 M_{1}+\left(M_{2}-M_{1}\right) / a$ as $t \rightarrow \infty$. Thus, (13) is satisfied if

$$
[(2 a-1) /(2 a)] M_{1}+[1 /(2 a)] M_{2}<1 / 2 .
$$

One can easily see that $\alpha /(2 \beta)<a^{2} /[(a+1)(2 a-1)]$ for every $a>1$, and hence the triangular region $\Delta_{1}$ in the $M_{1} M_{2}$-plane determined by (17) is contained in the triangular region $\Delta_{2}$ determined by (18). Furthermore, as $a \rightarrow \infty, \Delta_{1}$ shrinks to a point. In other words, (18) is milder than (17), and for a given kernel $C(t, s)$ satisfying (18), there is an $a>1$ such that (17) cannot be satisfied. For instance, if $c_{11}(t, s)=(3 s / 4)\left(t^{2}-s^{2}+1\right)^{-2}$ and $c_{12}=c_{21}=c_{22}=0$, then $M_{1}=3 / 8, M_{2}=0$, (18) is satisfied, and for $a \geq 4,(17)$ does not hold. Thus, solutions of (16) are uniform bounded by Theorem 1 while Theorem A fails to apply for all $a \geq 4$.

REMARK 4. Although condition (5) may be strong in some cases, it is often easier to apply than condition (13). Below, we give some stability criteria with conditions similar to the conditions of Theorems 1 and A except that the integrals involve the first argument of $C(t, s)$ rather than the second one.

Let $B(t)=B=$ constant in (6), and let $B$ be a stable matrix and $D$ be the unique positive definite symmetric matrix satisfying

$$
B^{T} D+D B=-I .
$$

Let $\alpha^{2}$ and $\beta^{2}$ be the smallest and largest eigenvalues of $D$. Then

$$
\alpha^{2}|z|^{2} \leq z^{T} D z \leq \beta^{2}|z|^{2} \text { for all } z \in R^{n} .
$$

Let $H(t, s)$ and $V=V(t, x(\cdot))$ be as defined in equations (8)-(9), and suppose that $\int_{t}^{\infty}|H(u, t)| d u$ and $\int_{t}^{\infty}|C(u, t)-K(u, t)| d u$ exist. Let $W(t, x(\cdot))=\left[V^{T} D V\right]^{1 / 2}+$ $\Phi(t, x(\cdot))$, where

$$
\Phi(t, x(\cdot))=\int_{0}^{t} \int_{t}^{\infty}\left\{\frac{1}{2 \beta}|H(u, s)|+\frac{1}{\alpha}|D[C(u, s)-K(u, s)]|\right\} d u|x(s)| d s .
$$

The derivative of $W(t, x(\cdot))$ along a solution $x(t)=x\left(t, t_{0}, \phi\right)$ of $(2)$ satisfies

$$
W^{\prime}(t, x(\cdot))=\left[V^{\prime T} D V+V^{T} D V^{\prime}\right] /\left[2\left(V^{T} D V\right)^{1 / 2}\right]+\Phi^{\prime}(t, x(\cdot)),
$$

where

$$
\begin{aligned}
\Phi^{\prime}(t, x(\cdot))= & \int_{t}^{\infty}\left\{\frac{1}{2 \beta}|H(u, t)|+\frac{1}{\alpha}|D[C(u, t)-K(u, t)]|\right\}|x| \\
& -\int_{0}^{t}\left\{\frac{1}{2 \beta}|H(t, s)|+\frac{1}{\alpha}|D[C(t, s)-K(t, s)]|\right\}|x(s)| d s .
\end{aligned}
$$

By (10) and (19)-(21)

$$
\begin{aligned}
W^{\prime}(t, x(\cdot))= & \left.-|V|^{2}+2 V^{T} \int_{0}^{t} D[C(t, s)-K(t, s)] x(s) d s\right\} /\left[2\left(V^{T} D V\right)^{1 / 2}\right] \\
& +\Phi^{\prime}(t, x(\cdot)) \\
\leq- & \frac{1}{2 \beta}|V|+\frac{1}{\alpha} \int_{0}^{t}|D[C(t, s)-K(t, s)]||x(s)| d s+\Phi^{\prime}(t, x(\cdot)) \\
\leq- & \frac{1}{2 \beta}|x|+\int_{0}^{t}\left\{\frac{1}{2 \beta}|H(t, s)|+\frac{1}{\alpha}|D[C(t, s)-K(t, s)]|\right\}|x(s)| d s \\
& +\Phi^{\prime}(t, x(\cdot)),
\end{aligned}
$$


(22)

$$
W^{\prime}(t, x(\cdot)) \leq\left\{\frac{1}{2 \beta}\left[-1+\int_{t}^{\infty}|H(u, t)| d u\right]+\frac{1}{\alpha} \int_{t}^{\infty}|D[C(u, t)-K(u, t)]| d u\right\}|x| .
$$

THEOREM 2. If $B$ is a stable matrix and $P$ is a positive constant such that

$$
\int_{t}^{\infty}\left|Z(u-t)[A(t)-B]+\int_{t}^{u} Z(u-s) C(s, t) d s\right| d u \leq P<1,
$$

then every solution of $(2)$ is $L^{1}[0, \infty)$.

ProOF. Let $K(t, s)=C(t, s)$. Then by $(22)-(23), W^{\prime}(t, x(\cdot)) \leq(P-1)|x| /(2 \beta)$ for all $t \geq t_{0}$. Thus,

$$
0 \leq W(t, x(\cdot)) \leq W\left(t_{0}, \phi\right)+[(P-1) /(2 \beta)] \int_{t_{0}}^{t}|x(s)| d s .
$$

This completes the proof.

THEOREM 3. Suppose $A(t)=A=$ constant, $A$ is stable, and $D, \alpha^{2}, \beta^{2}$ are as defined in (19)-(20). If there is a positive constant $M$ such that

$$
\int_{t}^{\infty}|D C(u, t)| d u \leq M<\alpha /(2 \beta)
$$

then every solution of $(2)$ is $L^{1}[0, \infty)$, and the zero solution is stable.

Proof. Let $B=A$ and $K(t, s) \equiv 0$. Then by (8), $H(t, s) \equiv 0$, and by (22)-(24)

$$
W^{\prime}(t, x(\cdot)) \leq\left[-\frac{1}{2 \beta}+\frac{1}{\alpha} \int_{t}^{\infty}|D C(u, t)| d u\right]|x| \leq\left(-\frac{1}{2 \beta}+\frac{M}{\alpha}\right)|x| .
$$

As $W(t, x(\cdot))$ is positive definite, the proof is complete.

REMARK 5. When $A(t)=A=$ constant and $C(t, s)=C(t-s)$, then (23) coincides with (ii) of Theorem 1 , and hence the conclusion of Theorem 2 is precisely the statement that the resolvent associated with

$$
x^{\prime}=A x+\int_{0}^{t} C(t-s) x(s) d s+f(t)
$$

is $L^{\mathbf{1}}[0, \infty)$. Furthermore, if $C \in L^{\mathbf{1}}[0, \infty)$, then, by Miller [6], the resolvent associated with (25) is $L^{1}$ if and only if the zero solution of

$$
y^{\prime}=A y+\int_{0}^{t} C(t-s) y(s) d s
$$

is u.a.s.

COROLlaRY 3. If $C \in L^{1}[0, \infty)$ and there is a stable matrix $B$ such that

$$
\int_{0}^{\infty}\left|Z(t)(A-B)+\int_{0}^{t} Z(t-u) C(u) d u\right| d t<1
$$

then the zero solution of (26) is u.a.s.

REMARK 6. Corollary 3 includes some of the results obtained in [3, pp. 159 162]. In fact, for $A=0$ and $B=-\lambda I,(27)$ reduces to (29) of [3] and, for $A$ stable and $B=A$, it reduces to (34) of [3]. Thus, Theorem 1 can also be considered as an extension of these results in [3] to the nonconvolution system (1). 


\section{REFERENCES}

1. T. A. Burton, Perturbed Volterra equations, J. Differential Equations 43 (1982), 168-183.

2. _ Periodicity and limiting equations in Volterra systems, Boll. Un. Mat. Ital. 4 (1985), 31-39.

3. R. Grimmer and G. Seifert, Stability properties of Volterra integrodifferential equations, J. Differential Equations 19 (1975), 142-166.

4. S. I. Grossman and R. K. Miller, Nonlinear Volterra integrodifferential systems with $L^{1}$ kernels, J. Differential Equations 13 (1973), 551-566.

5. J. K. Hale, Ordinary differential equations, Wiley, New York, 1969.

6. R. K. Miller, Asymptotic stability properties of linear Volterra integrodifferential equations, J. Differential Equations 10 (1971), 485-506.

Department of Mathematics, MurRay State University, MurRay, KentuCKy 42071 